\title{
Learned flavor preferences based on caloric outcome
}

\author{
RONALD MEHIEL and ROBERT C. BOLLES \\ University of Washington, Seattle, Washington
}

\begin{abstract}
Two experiments paired flavors with ethanol, sucrose, or ethanol and polycose solutions to investigate learning of flavor preferences by rats. When one solution offered a caloric advantage over the other, a preference was learned for its associated flavor. When one solution offered no caloric advantage over the other, no preference was learned for the associated flavors. Increasing the caloric value of the ethanol while holding the actual percentage of ethanol in solution constant fostered a preference for a flavor associated with the more dense solution. Recent reports involving ethanol intake are examined in light of this calorie-based learned-preference mechanism.
\end{abstract}

There is abundant evidence for learning about negative postingestional effects of a food and learning to avoid the flavor of that food. For nearly three decades (see Riley \& Clarke, 1977, for a bibliography), a copious liturature has been built on studies using a tasty solution or food paired with a sickness-inducing agent (usually lithium) in which animals learn to avoid the sickness-paired taste. This kind of learning is easy to get and hard to extinguish. It seems clear in taste-aversion learning that the taste of a particular food becomes associated with the organismic changes that result from ingestion of the food. This is true even when the food is not the cause of the sickness (e.g., when $x$-rays are used to induce illness; Garcia, Kimmeldorf, \& Koelling, 1955).

In contrast to the clear picture that has emerged with conditioned taste aversions, there is at best only sparse evidence for learning about the positive aspects of foods and the flavors associated with them. One line of evidence is in the area of learned taste preferences based on a "medicine effect." L.e Magnen, Marfaing-Jallet, and Miceli, (1980) described rats that were dependent upon alcohol and were exhibiting withdrawal symptoms. These rats were given saccharin paired with small doses of alcohol for 2,4 , or 8 days. Later, when tested for saccharin consumption, they displayed a positive linear relationship between the number of pairings and saccharin consumption. Le Magnen et al. concluded that a medicine effect was conditioning the learned preference. $\mathrm{Za}$ horik and Maier (1969) offered rats a flavor previously paired with recovery from thiamine deficiency and found a preference for that flavor over other flavors presented in various control conditions. Rozin (1967) showed that rats with experience of sickness tend to prefer familiar flavors over novel ones. That is, they display increased neophobia relative to controls. Zahorik, Maier, and Pies

\footnotetext{
We wish to thank Moncrief Smith and Hank Sampson for their editorial assistance in the preparation of this manuscript. The authors' mailing address is: Department of Psychology, University of Washington, Seattle, WA 98195.
}

(1974) took this neophobia interaction into account and in a well-designed study offered conclusive evidence that rats really do prefer flavors paired with recovery from illness. In a retardation type of experiment (Zahorik \& Bean, 1975), this medicine-effect mechanism was shown to increase the resistance of recovery-paired flavors to learned aversions.

Positive nutritional outcome seems a likely candidate for the support of learned taste preferences. What could be better for a foraging omnivore, than to have a learning mechanism that not only allows it to avoid food that makes it ill, but also to like food that provides calories? Few studies have addressed this issue. Bolles, Hayward, and Crandall (1981) offered rats flavored diets that differed in caloric density and found a preference for the flavor associated with the higher density diet. However, they used chalk as a dilutant, and it remains unclear whether the rats were tracking calories or simply avoiding the chalk. Hayward (1983) replicated the above findings with juvenile and adult rats, but again used chalk as a dilutant.

To avoid the possibility of conditioning an aversion to a dilutant in the present studies, we used solutions instead of solid food. In a pilot study, we paired orange and grape Kool Aid (General Foods) with either sucrose or saccharin and alternated the solutions daily for 4 days. We reasoned that since both sucrose and saccharin are readily consumed by the rat, flavors paired with them should become more acceptable. However, sucrose is a tasty food with caloric payoff whereas saccharin is tasty but inert. If flavors paired with calories are preferred, then a sucrose-paired flavor should be preferred over a saccharin-paired flavor.

For 4 days the rats alternated between sucrose and saccharin with balanced flavors attached; then they were given a two-bottle choice test between the Kool Aid flavors only. Every rat preferred the flavor paired with sucrose over the flavor paired with saccharin. However, as Figure 1 shows, consumption was not equal during conditioning trials, and the possibility exists that this differen- 


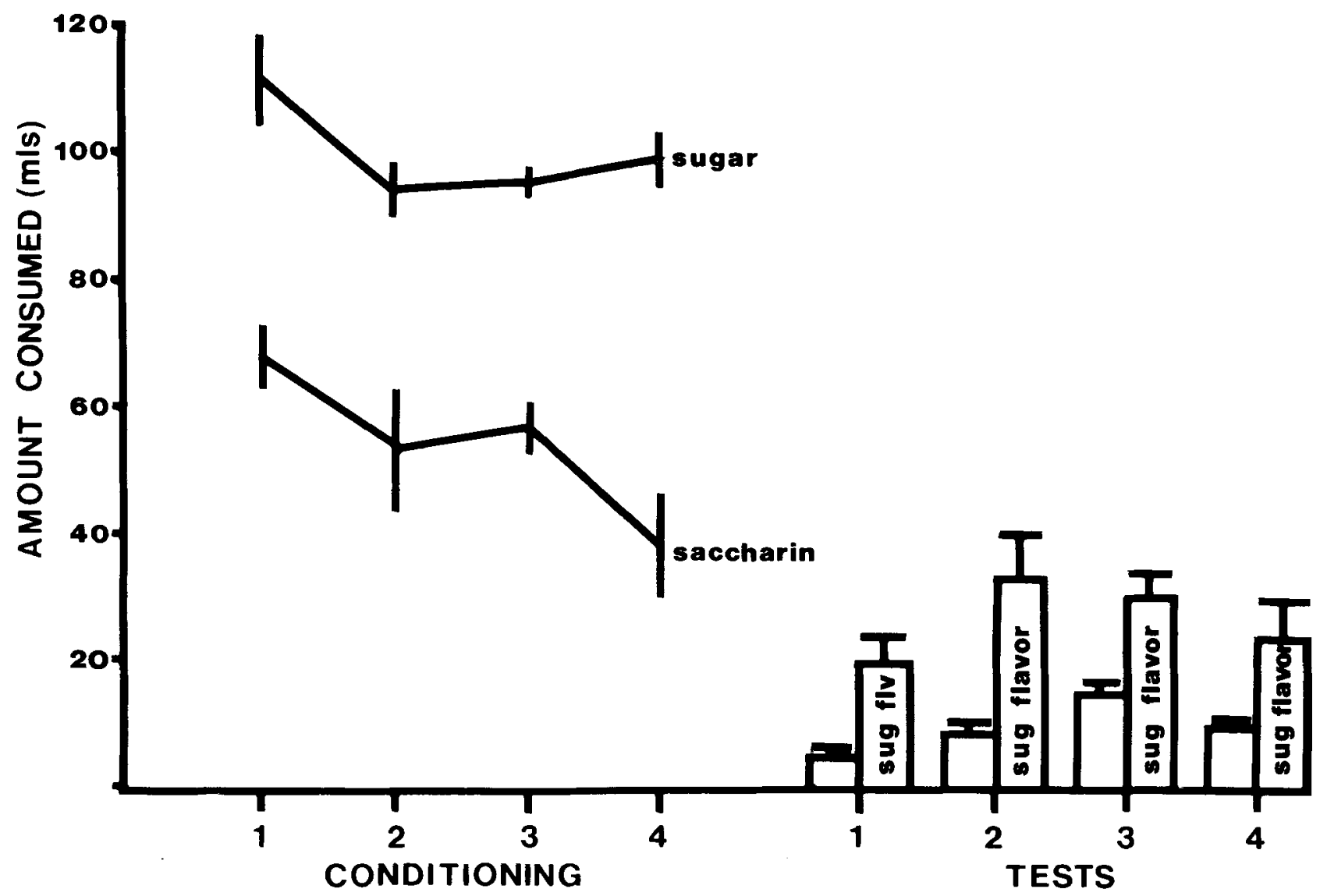

Figure 1. Mean daily intake and standard error for saccharin and sugar on conditioning days, and of associated flavors during tests.

tial consumption contributed to the direction of the learned preferences.

Arguing against the differential consumption possibility just considered are data presented by Holman (1975), who reported that a flavor paired with saccharin and available $60 \mathrm{~min}$ per day was not preferred over another flavor paired with saccharin and available only 5 min per day. In other words, amount of exposure had no effect on learned preference. His procedure was similar to ours in that the solutions were alternated daily. Even though his rats drank four to five times as much saccharin solution in the 60-min periods, no preference was learned for the attached flavor. A second experiment in the same paper showed that, in a two-bottle test, a flavor paired with a high concentration of saccharin is preferred over a flavor paired with a dilute concentration of saccharin. Thus, we can rule out the possibility that a learned aversion to the saccharin-paired flavor is responsible for our results. We have looked specifically for such an effect and have failed to find it.

Holman (1975) also showed that a flavor followed $30 \mathrm{~min}$ later by dextrose will be preferred to a flavor followed by saccharin, and he concluded that the caloric benefit of the dextrose was perhaps responsible for conditioning the preference. If the US is, indeed, the caloric benefit of the sugar, then rats should be able to learn a flavor preference based on calories even when not labeled by innately preferred sweetness. With this idea in mind, we performed a second pilot study in which another group of rats was subjected to the same alternating solution procedure, but $5 \%$ ethanol was substituted for sugar as the calorie source. Pairing ethanol with neutral flavors most typically produces a learned aversion for the neutral flavor (Cappell, LeBlanc, \& Endrenyi, 1972; Cunningham, 1978; Lester, Nachman, \& Le Magnen, 1970). But in a two-bottle test, the rats once again preferred the flavor associated with the calorie source over the flavor paired with saccharin.

One problem with using ethanol as a food, however, is that it is difficult to separate its pharmacological properties from its nuturitional properties. In a third pilot study, we again used ethanol and saccharin in an alternating conditioning procedure, but there were four subgroups of rats which differed in the concentration of ethanol they received. Concentrations ranged from $5 \%$ to $12.5 \%$. In testing, the two lower concentration groups preferred the flavor paired with ethanol, whereas the two higher groups did not. Interestingly, when given a two-bottle test between plain ethanol and saccharin, all of the groups preferred the saccharin. 
There was abundant evidence that various aversive pharmacological agents, including ethanol, could support conditioned taste aversion (Cunningham, 1978; Lester, et al., 1970). It would not seem that the pharmacological effect could be positively reinforcing because it was not clear that, at a daily dose of about $11 \mathrm{~g} / \mathrm{kg}$, blood alcohol would ever be high enough to produce much of a pharmacological effect (Lester \& Greenberg, 1952). Nor was it plausible to think of the taste of ethanol itself as being a reinforcer, because under no condition was the ethanol taste preferred. Since saccharin was invariably preferred over ethanol, saccharin-paired flavors should always be preferred over ethanol-paired flavors, if taste alone were the major factor in reinforcement. It seems likely that the conditioned taste preference was reinforced by the caloric content of the ethanol, but that in the higher percentage groups the aversive taste of the ethanol prevented this conditioning. On the other hand, it was possible that at lower concentrations of ethanol, the pharmacological effects were reinforcing, whereas at the higher concentrations they were not.

In summary, our three pilot studies showed two things. Flavors paired with sugar and flavors paired with low concentrations of ethanol were preferred over flavors paired with saccharin. High doses of ethanol, however, would not support a learned flavor preference. This failure of conditioning at higher concentrations could be due to the aversive taste of the ethanol or to the greater pharmacological effects. It was still not certain what the reinforceing US was in the situations in which an ethanol-associated flavor came to be preferred. The positive reinforcer had not been satisfactorily identified. The following two experiments were performed to clarify this situation.

\section{EXPERIMENT 1}

The present experiment compared isocaloric solutions of sugar and $5 \%$ ethanol, and their effect on conditioning a preference for neutral flavors, with sugar and 5\% ethanol solutions that were not isocaloric (the sugar solution had fewer calories). If it was the pharmacological properties of ethanol ingestion that supported this conditioned preference, then rats in both isocaloric and nonisocaloric groups should prefer the ethanol-paired flavor over the sugar-paired flavor. If, however, the caloric properties of ethanol supported the conditioned preference, then a preference for the ethanol-paired flavor should emerge only in the nonisocaloric condition, the condition in which the ethanol solution contained more calories than did the sugar solution.

\section{Method}

Subjects. Sixteen naive male rats served as subjects. Eight were Long-Evans rats and eight were Wistars. (The two strains were used to see if there were any strain differences in ethanol consumption and in flavor conditioning.) The rats' mean weight was $385.5 \mathrm{~g}$, and they were 120 days old at the start of the experiment. They were housed individually and were maintained on a natural $12 / 12$ $\mathrm{h}$ light/dark cycle. Five days prior to the beginning of condition- ing, all rats were placed on a regimen of $12 \mathrm{~g}$ of Purina Rat Chow per day and ad-lib water. All conditioning and testing was done in the home cages, which were hanging metal cages with grid floors.

Procedure. The subjects were divided randomly into two groups, each group containing four Long-Evans and four Wistar rats. The groups differed only in the concentration of sugar they received in the conditioning solutions. On the first day, all rats got a $5 \%$ ethanol $(w / v)$ solution. Half of each group got ethanol paired with grape, and half got ethanol paired with orange. The flavors were prepared from unsweetened Kool Aid (General Foods), 1 packet to 2 liters of tap water. The solutions were on the cages for $24 \mathrm{~h}$. The next day, the bottles were removed and measured and the 12$\mathrm{g}$ daily ration of chow was presented. Rats in the isocaloric group (7.6\% sugar $w / v)$ were then presented with an amount of sugar solution equal to what they had consumed of the ethanol. In this way, they could not get more calories from the sugar than from the ethanol. Rats in the low-calorie group ( $1 \%$ sugar w/v) were likewise given an amount equal to their consumption of the ethanol. During conditioning, this yoking procedure ruled out any differential consumption of the ethanol and sugar solutions due to an innate preference for the sugar. Conditioning proceeded in this alternating yoked sequence for 8 days, so that each rat had 4 days of exposure to ethanol and 4 days of exposure to sugar, with the grape and orange counterbalanced.

Testing. Following the last day of conditioning, all rats were allowed ad-lib access to chow and water for $72 \mathrm{~h}$. This was followed by a $24-h$ period of water only and then a $1-h$ preference test between orange and grape flavors in water without ethanol or sugar. Following the first test, food was returned for ad-lib access for $24 \mathrm{~h}$, followed by another 24 -h water-only period and a second preference test. The second test, which again lasted $1 \mathrm{~h}$, was a choice test between the two conditioning solutions in their entirety. Three more days of ad-lib chow, another 24-h deprivation period, and a third test followed. On the morning of the third test, all rats were intubated by gavage with $5 \mathrm{ml}$ of a $37 \% \mathrm{w} / \mathrm{v}$ sugar solution. This was equivalent to about $20 \mathrm{kcal}$ and was isocaloric to the mean intake of sugar solution in the second test. Twenty minutes after gavage, a 1-h test between the orange and grape flavors ensued. After another 3 days of ad-lib chow and water followed by a 24-h deprivation period, a fourth test, lasting $4 \mathrm{~h}$, was conducted again between flavors only.

\section{Results}

Intake during conditioning for both the isocaloric group and the low-calorie groups is shown in Figure 2. All rats in the isocaloric group consumed all available sugar solution. Their intake of ethanol and sugar rose significantly over days $[F(1,4)=21.83, p<.01]$. In the low-calorie group, the trend for ethanol consumption was the same; in fact, the groups never differed significantly in ethanol intake. However, sugar-solution consumption for the lowcalorie group failed to match ethanol consumption, starting on the second presentation of sugar solution. This trend continued to the end of conditioning, with the rats leaving more sugar solution in the bottles every day.

Mean intakes in the tests are shown in Figure 3. The rats in the isocaloric group displayed a slight, but insignificant, preference for the sugar-paired flavor in the first test $[F(1,7)<1]$. The low-calorie group displayed a significant preference for the ethanol-associated flavor $[\mathrm{F}(1,7)=5.55, \mathrm{p}<.05]$.

The results of the second test, in which the conditioning solutions were offered, yielded a highly significant preference for the sugar solution in the isocaloric group 


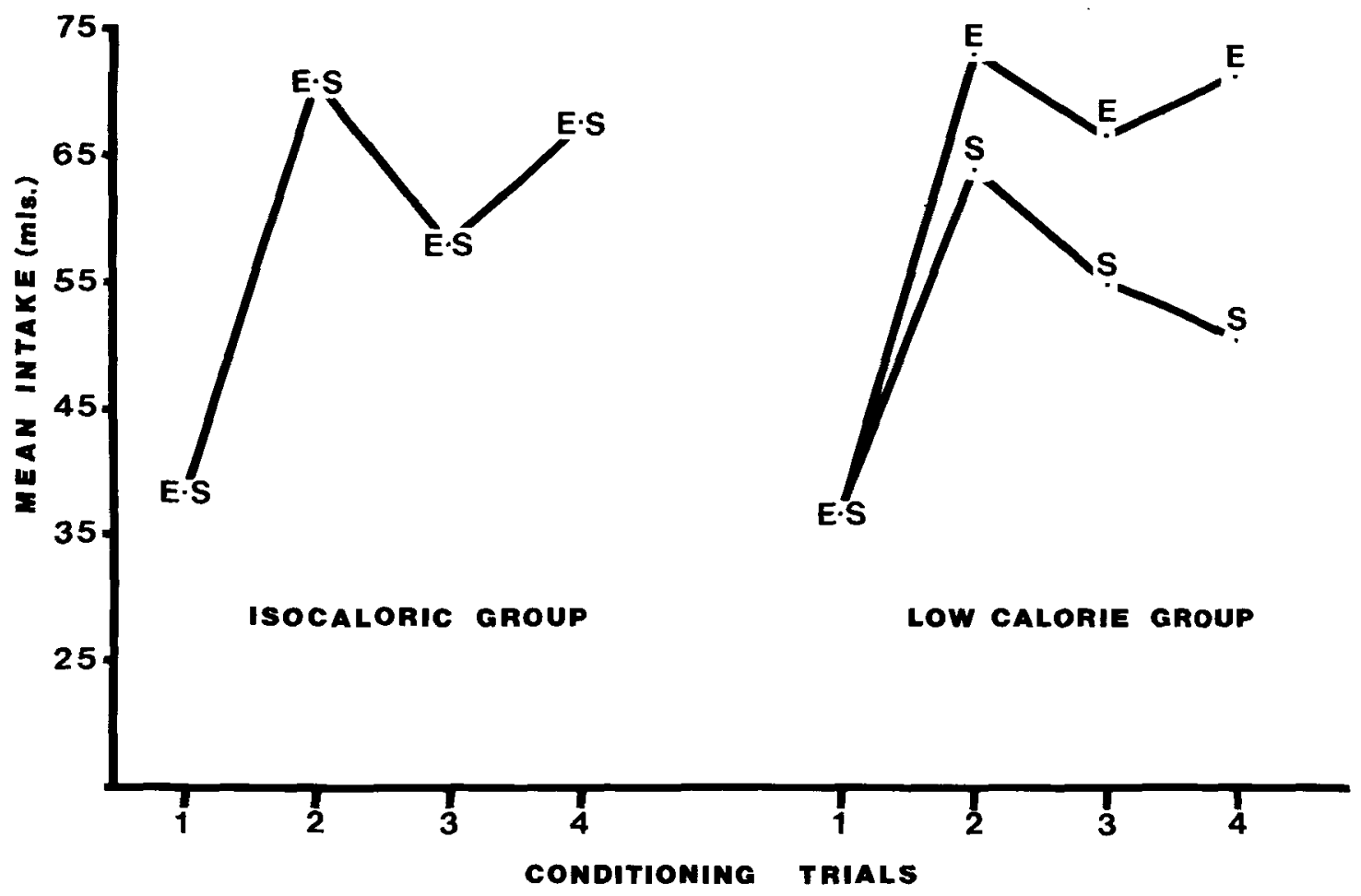

Figure 2. Mean daily intake of ethanol and sugar on conditioning days in Experiment 1 for isocaloric and low-calorie groups.

$[F(1,7)=228.7, p<.01]$, but a significant preference for the ethanol solution in the low calorie group $[F(1,7)$ $=10.3, \mathrm{p}<.05]$.

The third test, in which the rats were first gavaged with sugar, yielded no preference for either flavor. Consumption during the test was depressed for all rats and no differences were observed.

The last test was a replication of the first test, except that it lasted $4 \mathrm{~h}$ rather than $1 \mathrm{~h}$. Once again, the rats in the isocaloric group showed a nonsignificant preference for the sugar-paired flavor, whereas the rats in the lowcalorie group had a significant preference for the ethanolassociated flavor $[F(1,7)=.49$ and 26.01]. analysis of variance using strains as a factor showed no differences in consumption or testing between Long-Evans and Wistar rats.

\section{Discussion}

The results of Experiment 1 support the idea that a calorically based unconditioned stimulus is responsible for the learned preferences we have demonstrated so far. In the isocaloric group, no preference for the ethanol-paired flavor was conditioned. More importantly, there was no conditioned preference for the sugar-paired flavor. Our pilot work showed that sugar or ethanol will support a learned preference for an attached flavor when juxtaposed with saccharin. In Experiment 1, however, the solutions offered equal caloric benefit. Unless we are willing to assume that the sweetness of sugar and the pharmacologi- cal effects of a $5 \%$ ethanol solution are precisely equal in their ability to condition a flavor preference with this paradigm, we are forced to look to caloric value or taste alone as the US. If pharmacological effects were responsible, then both the isocaloric and the low-calorie groups should have learned a preference for the ethanol-associated flavor. The results for the low-calorie groups are essentially a replication of our pilot studies. A $1 \%$ sugar solution offers only $.038 \mathrm{kcal} / \mathrm{ml}$ and is probably not worth the energy to consume it. It is, however, above the threshold for the taste of sugar as reported in previous research (Richter \& Campbell, 1940) and would presumably be preferred over a completely nonnutritive solution such as water. Note that the rats in the isocaloric group preferred the sugar solution to the ethanol solution when offered both in the second test. This preference must be based on taste alone, but taste alone fails to support a learned preference for the attached flavor.

\section{EXPERIMENT 2}

If the pharmacological effects of ethanol are responsible for the learning we have demonstrated here, then in the previous experiment we should have seen in both groups a preference for the flavor associated with ethanol. However, another interpretation of the results may be that pharmacological effects of ethanol and innate preferences for sweetness are equal in their ability to condition a preference for an associated flavor. We have seen that 


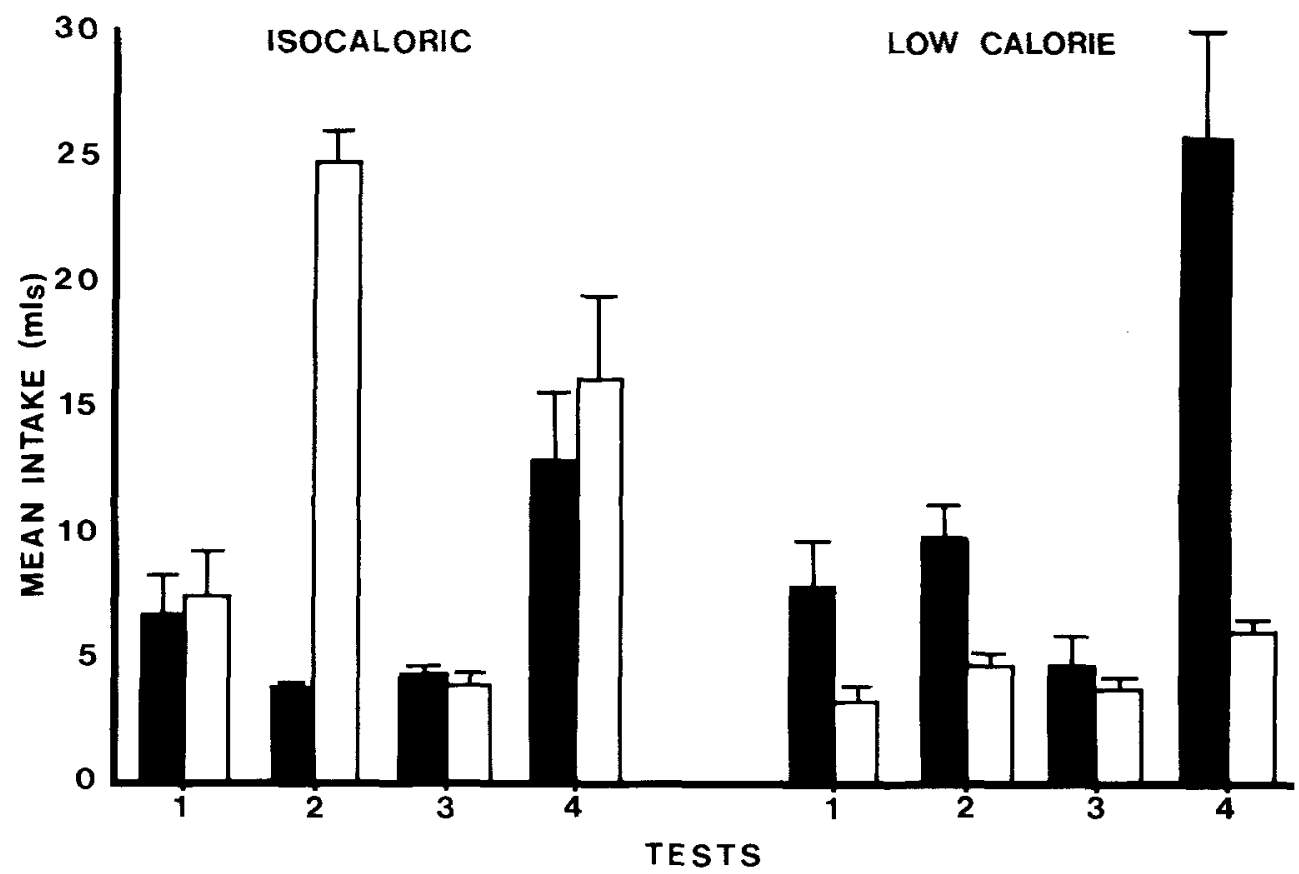

Figure 3. Mean intake during testing in Experiment 1 for isocaloric and low-calorie groups. T1 = flavors only; $\mathbf{T} 2=$ conditioning solutions; $\mathbf{T 3}=$ gavage and flavors only; $\mathbf{T} 4=$ flavors only. Light bars $=$ sugar, dark bars = ethanol.

sweetness alone will not serve as an unconditioned stimulus in this paradigm; however, we still cannot rule out the pharmacological effects of the ethanol. If the drug effects, and not the calories, are primarily reinforcing the conditioned taste preferences we have observed, then when rats are presented with $5 \%$ ethanol every day in our alternating conditioning procedure but one solution has twice the caloric density of the other, no preference for the attached flavors should be learned. This hypothesis was tested in Experiment 2.

\section{Method}

Subjects. Twelve naive male Long-Evans rats served as subjects. Their mean weight was $365.4 \mathrm{~g}$, and they were 90 days old at the start of the experiment. They were housed individually as in the previous experiments.

Solutions. Lime and orange Kool Aid were used as flavor labels. They were mixed in the same proportions as in the previous experiment. Once again, a 5\% ethanol solution was used; however, its caloric value was doubled by the addition of $7.6 \%(w / v)$ of Polycose (Ross Laboratories; Columbus, Ohio). Polycose is a tasteless powder derived from hydrolized corn starch and is isocaloric with sucrose.

Procedure. The rats were randomly assigned to two groups of six. The groups differed only in the flavor attached to the highcalorie ethanol $(\mathrm{E}+)$. For 3 days prior to the start of conditioning and throughout conditioning, all rats were restricted to $10 \mathrm{~g} \mathrm{of} \mathrm{Pu-}$ rina Rat Chow per day. Water was never available while the experimental solutions were on the cages. On the first day of conditioning, all rats received $100 \mathrm{ml}$ of the plain $5 \%$ ethanol solution with the appropriate flavors mixed in. On the next day, the bottles were removed and measured, the rats were fed, and the E+ solution was presented with the other flavor mixed in. Each rat received only enough solution to enable it to match its previous day's con- sumption. This yoking procedure again ensured that the development of a flavor preference could not be based upon differential intake. Conditioning proceeded in this manner for 8 days so that each rat had 4 days of exposure to each flavor and each ethanol solution. On the day following the end of conditioning, all rats were fed $10 \mathrm{~g}$ of chow and offered water ad lib.

Testing. On the next day, a two-bottle choice test between lime and orange with no ethanol or polycose was run prior to the day's chow ration. Since the rats had developed the habit of eating the full $10-\mathrm{g}$ ration in about $1 \mathrm{~h}$, they were effectively $23-\mathrm{h}$ deprived at the start of the test. Following the test, the $10 \mathrm{~g}$ were fed and water was returned ad lib. On the next day, again prior to feeding, a two-bottle test was run between the two flavors, but this time both flavored solutions contained $5 \%$ ethanol and $3.8 \%$ polycose. The solutions were isocaloric but differed in flavor; we wanted to see if the rats would track the $\mathrm{E}+$ flavor even when the test solutions were isocaloric.

\section{Results and Discussion}

Intake during conditioning and testing is shown in Figure 4. The rats showed suprisingly little neophobia, drinking over $65 \mathrm{ml}$ of ethanol on the first day. All of the $\mathrm{E}+$ solutions were consumed by every rat on every day they were available. Consumption on the fourth block of trials was significantly greater than on the previous three $[F(1,11)=16.8, p<.05]$. In the first test, the $E+-$ paired flavor was preferred over the ethanol-paired flavor about 3 to 1 . This difference was significant $[F(1,11)=$ $4.9, \mathrm{p}<.05]$. The preference was again evident in the isocaloric test $[\mathrm{F}(1,11)=24.39, \mathrm{p}<.01]$.

In both tests, 10 of the 12 rats preferred the flavor paired with the more caloric ethanol over the flavor paired with ethanol only. Since flavors paired with ethanol are 


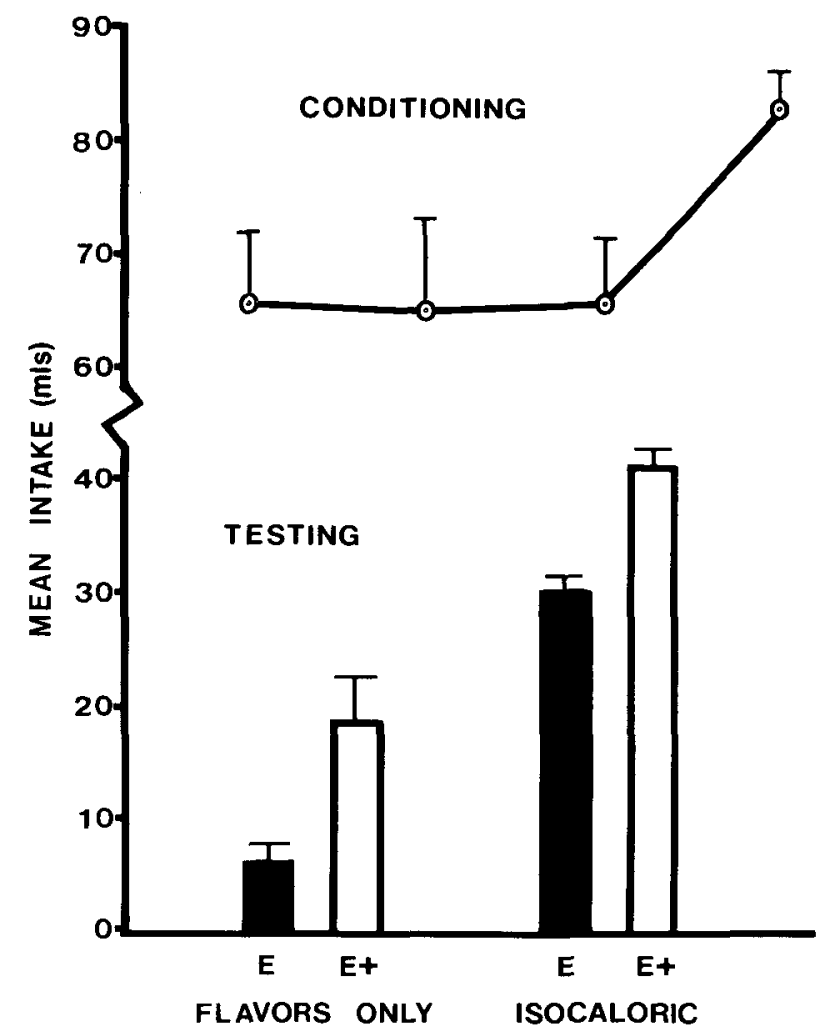

Figure 4. Mean intake during conditioning and testing in Experiment 2.

preferred over flavors paired with poor calorie sources (pilot data and Experiment 1), but are not preferred when the alternative flavor is paired with calorie sources that are equal to or greater than ethanol (Experiments 1 and 2 ), perhaps it is the caloric density of the solution that is responsible for the learned preferences demonstrated here.

\section{GENERAL DISCUSSION}

There is mounting evidence for learned preferences based on nutritional outcome. Sherman, Hickis, Rice, Rusiniak, and Garcia (1983) found preferences for a flavor associated with a low dose of gavaged ethanol, no difference with a medium dose, and an aversion with a high dose relative to a flavor associated with water. Moreover, when the ethanol provided no caloric advantage (when it was juxtaposed against a gavage of isocaloric glucose), no preference was learned. Although our procedure differs from that of Sherman et al., their results are in agreement with those of our Experiment 1.

A number of studies have investigated the relationship between ethanol intake and caloric restriction of diet. Probably the best example of the rat's behavior when ethanol is available and food is restricted was reported by Westerfeld and Lawrow (1953). They made $10 \%$ ethanol available to three groups of rats that differed on the basis of whether they were restricted to $100 \%, 75 \%$, or $50 \%$ of their ad-lib chow intake. Over a period of 15 weeks, all groups showed a gain in weight. The rats in the $50 \%$ and $75 \%$ groups made up the caloric deficit by drinking more ethanol. When the availability of chow was reversed, ethanol intake likewise reversed.

Hausmann (1932) investigated the effect of food restriction on the intake of water, alcohol, and sugar solutions in various concentrations and concluded that the rat regulates its consumption of ethanol so that the same absolute amount of ethanol is ingested no matter what the concentration, and that the increase in calories from alcohol was always offset by an appropriate reduction in chow intake. He reported the same calorie regulation when sugar solution was available. In a similar experiment, Lester and Greenberg (1952) showed that the rat will give up ethanol intake to drink fat, sucrose, or saccharin. In addition, when the solid diet was increased in caloric density, ethanol consumption likewise decreased.

Crawford and Baker (1982), in an investigation of the medicine-effect conditioning of flavor preferences, had control groups that received small gavaged doses of ethanol paired with a saccharin taste stimulus. They were surprised to find no aversion learning for the saccharin in these low-dose rats. Instead of learning an aversion to the ethanol-paired flavor, their rats learned a preference for it.

Wayner, Barone, and Jolicoeru (1978) forced groups of rats to drink $1.7 \%$ sodium chloride for a period of 117 days. One control group had various concentrations of ethanol paired with the salt for $\mathbf{5 5}$ days, and another group had citric acid paired with salt for the same period. During the remainder of the 117-day period, the ethanol group drank much more sodium chloride solution than did the other groups. Wayner et al. concluded that the marked increase of salt-solution intake was attributable specifically to the prolonged ingestion of ethanol and to withdrawal symptoms. However, another interpretation of their results is that a preference for the salt flavor was learned through association with the caloric benefit of the ethanol missing from their other groups.

In conclusion, what we offer here is evidence that rats can come to prefer an initially aversive ethanol solution, and that the reinforcer for that learning is the calories in the ethanol. This learning mechanism, in which postingestional effects are associated by the animal with the flavor consumed, has been demonstrated in a variety of paradigms. These results offer a new and simpler explanation for a number of previously reported results, especially those investigating an animal model of alcoholism.

\section{REFERENCES}

Bolles, R. C., Hayward, L., \& Crandall, C. (1981). Conditioned taste preferences based on caloric density. Journal of Experimental Psychology: Animal Behavior Processes, 7, 59-69.

Cappell, N., LeBlanc, A. E., \& Endrenyi, L. (1972). Effects of chlordiazepoxide and ethanol on the extinction of a conditioned taste aversion. Physiology \& Behavior, 9, 167-169. 
Crawford, D., \& BaKer, T. B. (1982). Alcohol dependence and taste mediated learning in the rat. Pharmacology, Biochemistry and Behavior, 16, 253-261.

Cunningham, C. L. (1978). Alcohol interacts with flavor during extinction of conditioned taste aversion. Physiological Psychology, 6 , 510-516.

Garcia, J., Kimmeldorf, D. J., \& Koelling, R. A. (1955). Conditioned aversion to saccharin resulting from exposure to gamma radiation. Science, 122, 157-158.

HaUsmanN, M. F. (1932). The behavior of albino rats in choosing food and stimulants. Journal of Comparative Psychology, 13, 279-309.

HAYWARD, L. (1983). The role of oral and postingestional cues in the conditioning of taste preferences based on differing caloric density and caloric outcome in weanling and mature rats. Animal Learning \& Behavior, 11, 325-331.

Holman, E. W. (1975). Immediate and delayed reinforcers for flavor preferences in rats. Learning and Motivation, 6, 91-100.

Le Magnen, J., Marfaing-Jallat, P., \& Miceli, D. (1980). A bioassay of ethanol dependence in rats. Pharmacology, Biochemistry and Behavior, 12, 707-709.

Lester, D., \& GreenberG, L. A. (1952). Nutrition and the etiology of alcoholism: The effect of sucrose, saccharin and fat on the selfselection of ethyl alcohol by rats. Quarterly Journal of Studies on Alcohol, 13, 553-560.

Lester, D., Nachman, M., \& Le Magnen, J. (1970). Aversive conditioning by ethanol in the rat. Quarterly Journal of Studies on Alcohol, 31, 578-586.

Richter, C. P., \& CAMPBelL, K. H. (1940). Taste thresholds and taste preferences of rats for five common sugars. Journal of Nutrition, 20, $31-46$.
Riley, A. L., \& Clarke, C. M. (1977). Conditioned taste aversions: A bibliography. In L. M. Barker, M. R. Best, \& M. Domjan (Eds.), Learning mechanisms in food selection. Waco, TX: Baylor University Press.

RozIN, P. (1967). Specific aversions as a component of specific hungers. Journal of Comparative and Physiological Psychology, 64, 237-242.

Sherman, J. E., Hickis, C. F., Rice, A. G., Rusiniak, K. W., \& GarCIA, J. (1983). Preferences and aversions for stimuli paired with ethanol. Animal Learning \& Behavior, 11, 101-106.

WAYNer, M. J., Barone, F. C., \& Jolicoeru, F. B. (1978). Effects of ethyl alcohol on forced consumption of an acclimated saline solution. Pharmacology, Biochemistry and Behavior, 8, 417-420.

WESTERFELD, W. W., \& LAWROW, M. S. (1953). The effect of caloric restriction and thiamine deficiency on the voluntary consumption of alcohol by rats. Quarterly Journal of Studies on Alcohol, 14, 378-384.

ZAHORIK D. M., \& BEAN, C. A. (1975). Resistance of "recovery" flavors to later association with illness. Bulletin of the Psychonomic Society, 6, 309-312.

ZAHORIK D. M., \& MAIER, S. F. (1969). Appetitive conditioning with recovery from thiamine deficiency as the unconditioned stimulus. Psychonomic Science, 17, 309-310.

Zahorik, D. M., Maier, S. F., \& Pies, R. W. (1974). Preferences for tastes paired with recovery from thiamine deficiency in rats: Appetitive conditioning or learned safety. Journal of Comparative and Physiological Psychology, 87, 1083-1091.

(Manuscript received February 21, 1984; revision accepted for publication August 17, 1984.) 